\title{
Satisfação dos usuários com a assistência de saúde no estado de Pernambuco, Brasil, 2005
}

\author{
Health care user satisfaction in Pernambuco State, Brazil, 2005
}

Giselle Campozana Gouveia ${ }^{1}$

Wayner Vieira de Souza ${ }^{1}$

Carlos Feitosa Luna ${ }^{1}$

Célia Landmann Szwarcwald ${ }^{2}$

Paulo Roberto Borges de Souza Júnior ${ }^{2}$

${ }^{1}$ Centro de Pesquisas Aggeu Magalhães, Fundação Oswaldo Cruz. Campus da Universidade Federal de Pernambuco. Av. Moraes Rego $s / n^{\circ}$, Cidade Universitária. 50670-420 Recife PE.

giselle@cpqam.fiocruz.br

${ }^{2}$ Centro de Informação

Científica e Tecnológica

Fundação Oswaldo Cruz.
Abstract Researching the users' satisfaction is a key task for management. With the Project for Strengthening the Evaluation Capacity of the State, the Pernambuco Health Secretariat has defined to evaluate the health system performance as priority. The current study aimed to know the factors associated with the users' satisfaction of the Pernambuco health system. It was an evaluative study based on the results obtained in a population survey carried out on 2005 in Pernambuco. The data analysis considered the use of multivariate methods and the satisfaction as dependent variable. The main analyzed aspects were the professionals quality, the health services quality and the health system resolubility. The analysis has revealed that the waiting time for health care and the availability of medicines were the factors with the lowest percentage of users' satisfaction, what generate reflections about if the access has been actually related to the offering of health services, adequate or not to the people demands and the pharmaceutical assistance policy in the state. In conclusion, it was possible to obtain a more complete overview about how the health system of Pernambuco has attended the people expectations by the users perspectives.

Key words Satisfaction, Health system, Factorial analysis, Evaluation
Resumo Pesquisar a satisfação dos usuários é tarefa fundamental para a gestão. Com o Projeto de Fortalecimento da Capacidade Avaliativa do Estado, a Secretaria de Saúde de Pernambuco identificou como demanda avaliar o desempenho do sistema de saúde no estado. O objetivo deste artigo é conhecer fatores associados à satisfação dos usuários do sistema de saúde pernambucano. Realizou-se estudo avaliativo a partir dos resultados obtidos num inquérito populacional com dados coletados em 2005 no estado de Pernambuco. A análise foi executada com emprego de métodos multivariados tendo a satisfação como variável dependente. Os principais aspectos analisados foram: qualidade dos profissionais, qualidade dos serviços de saúde e a resolutividade do sistema. As análises revelaram que o tempo de espera até o atendimento e a disponibilidade de medicamentos foram os fatores que geraram menores percentuais de satisfação dos usuários, o que leva a refletir se o acesso está de fato relacionado à oferta de serviços adequados às necessidades da população ou não e à política de assistência farmacêutica no estado. Concluindo, obteve-se um panorama mais completo sobre a forma como o sistema de saúde pernambucano tem respondido às expectativas de sua população na ótica dos usuários.

Palavras-chave Satisfação, Sistema de saúde, Análise fatorial, Avaliação 


\section{Introdução}

Nos últimos anos, os gestores e administradores do setor saúde têm enfatizado o papel da avaliação de desempenho das ações e dos programas de saúde, com o objetivo de garantir a qualidade da atenção e subsidiar decisões que atendam às reais necessidades da população ${ }^{1}$.

No contexto da qualidade em saúde, avaliar é diagnosticar uma realidade a fim de nela intervir para que os diferentes serviços de saúde cumpram padrões mínimos de qualidade ${ }^{2}$.

Malik e Schiesari ${ }^{3}$ afirmam que qualquer discussão a respeito de qualidade traz implícita ou explícita a noção de avaliação. Citam, ainda, Donabedian $^{4}$, quando referem três dimensões para a qualidade dos serviços de saúde: "o desempenho técnico", "as relações interpessoais" e "as amenidades".

A percepção dos usuários tem sido cada vez mais utilizada como uma medida importante na avaliação da qualidade dos cuidados em saúde. Para alguns autores ${ }^{5,6}$, a percepção dos cuidados é influenciada pela expectativa do usuário, bem como pelos cuidados recebidos de fato.

Alguns autores ${ }^{6}$ afirmam que os inquéritos de satisfação não refletem, necessariamente, as variações na forma como as pessoas estão realmente sendo tratadas pelo sistema de saúde, pois em pesquisa realizada em cinco países os valores encontrados nas taxas de satisfação foram menores para usuários dos Estados Unidos, que relataram ter gastado mais tempo com o atendimento médico em sua última consulta, no entanto os valores encontrados nas taxas de satisfação foram menores para esse país. Em outro estudo realizado em dezessete países ${ }^{7}$, foi revelado que os níveis de satisfação dos usuários se mostravam superiores em populações pobres comparados com as populações mais ricas, o que reforça a tese de que a percepção dos cuidados é influenciada pela expectativa do usuário.

Para alguns autores ${ }^{6,8}$, a responsividade é um conceito que reflete a real forma como um indivíduo é atendido nas suas necessidades de saúde pelos sistemas de saúde, portanto, espera-se que as avaliações de desempenho reflitam a realidade das experiências pessoais em termos de atendimento e cuidados em saúde, além de revelar as formas de interações entre os indivíduos e o sistema.

O uso dos serviços de saúde depende de determinantes individuais e necessidades de saúde, como a predisposição individual que influencia a capacidade de utilização destes, e as necessidades representam o determinante mais próximo da utilização deles ${ }^{9}$.

Para Trad e Bastos ${ }^{10}$, abordar a satisfação dos usuários implica trazer um julgamento sobre características dos serviços, portanto sobre sua qualidade. Assim, a perspectiva do usuário fornece informação para completar e equilibrar a qualidade dos serviços. Para Esperidião e Trad ${ }^{11}$, a satisfação envolve algumas dimensões ligadas aos aspectos dos serviços, tais como acesso, qualidade, estrutura física e organizacional, aspectos inerentes à relação médico-paciente etc.

Já para Stenzel et al. ${ }^{12}$, a satisfação resulta de juízos de diversos atributos, sendo a resolubilidade da demanda um deles. Vaistman et al. ${ }^{13}$ referem que a percepção dos usuários quanto às práticas de saúde nos serviços se desenvolve pela articulação de, pelo menos, quatro dimensões: a subjetividade individual, a cultura da sociedade, a rede de relações instituídas e a situação em determinado contexto ou a experiência imediata vivida.

A qualidade em serviços públicos e, mais concretamente, nos serviços de saúde é um desafio aos seus gestores, técnicos e todos os outros profissionais que têm capacidades que, se bem geridas, podem originar produtos aptos a satisfazer as necessidades dos seus usuários ${ }^{2}$.

Segundo Ferri et al. ${ }^{14}$, as práticas de saúde envolvem muitas considerações para que tenham como produto final o cuidado esperado, no qual está implícito que nos serviços ocorra: acolhimento, relações de responsabilidade, autonomia dos sujeitos envolvidos, necessidades de saúde, resolubilidade, compromisso, políticas públicas, enfim, a integralidade.

Considerando que a utilização dos serviços de saúde é produto da interação entre usuários, profissionais de saúde e serviços de saúde e que um dos principais problemas para que essa interação ocorra é a forma como o usuário consegue acessar os serviços, o acesso e o acolhimento são elementos essenciais do atendimento para que se possa incidir efetivamente sobre o estado de saúde do indivíduo e da coletividade ${ }^{15}$.

Cabe aqui destacar que o contexto brasileiro indica alguns fatores que podem influenciar a satisfação dos usuários, como a estrutura e a organização do sistema de serviços de saúde e modelos assistenciais vigentes no país. Para Esperidião e $\operatorname{Trad}^{11}$, o acesso é um atributo dos serviços bastante valorizado pelos usuários. Segundo essas autoras, o usuário de serviços públicos faz parte de populações que vivem em condições socioeconômicas precárias e que buscam os serviços de forma assistencial, interessado mais 
por um padrão mínimo de sobrevivência do que pela própria qualidade dos serviços.

Sabendo-se que pesquisar a respeito da satisfação dos usuários é uma tarefa fundamental para a gestão dos serviços, no ano de 2004, com o Projeto de Fortalecimento da Capacidade Avaliativa do Estado, a Secretaria de Saúde de Pernambuco (SES-PE) identificou como uma demanda urgente avaliar o desempenho do sistema de saúde no estado, na perspectiva do usuário.

Para otimizar as estratégias de desenvolvimento da capacidade técnica e contribuir com a implementação dos Planos Estaduais, foram contratados Centros Colaboradores que prestam assessoria permanente às Secretarias Estaduais de Saúde. Foram realizadas duas pesquisas avaliativas de interesse da SES-PE: Avaliação do Projeto Nascer e Pesquisa Mundial de Saúde-Atenção Básica (PMS-AB).

Pesquisa similar foi realizada em alguns municípios do Brasil nas regiões Sudeste e Norte, em 2005, com amostras representativas de estados apenas em Pernambuco e Rio de Janeiro ${ }^{1}$.

O instrumento utilizado na Pesquisa Mundial de Saúde (PMS) brasileira ${ }^{16}$ foi adaptado para avaliação da atenção básica em saúde. Alguns módulos do instrumento original foram mais detalhados. Salienta-se que, na PMS-AB, todo o módulo de resposta do Sistema de Saúde foi mantido na íntegra, como o da PMS brasileira.

A análise da PMS-AB deve aprofundar o conhecimento sobre o desempenho da atenção básica em saúde no estado, bem como, neste estudo, objetiva conhecer os fatores que estão associados à satisfação dos usuários do sistema de saúde pernambucano.

\section{Métodos}

Trata-se de um estudo avaliativo de corte transversal, a partir dos resultados obtidos num inquérito populacional de âmbito estadual. A análise foi realizada para o ano de 2005, em todo o estado de Pernambuco.

Foi incluída neste estudo uma amostra probabilística de todos os indivíduos com idade igual ou acima de 18 anos, que se internaram por 24 horas ou mais, ou utilizaram serviços ambulatoriais de saúde no último ano, para o período referente à coleta de dados.

A amostra foi calculada com base no cadastro municipal do Censo Demográfico de 2000 do Instituto Brasileiro de Geografia e Estatística (IBGE). Foram selecionados quarenta setores censitários em 27 municípios do estado. A situação (urbano ou rural) e o porte do município (até 50.000; 50.000 até 400.000; 400.000 + habitantes) estratificaram explicitamente as unidades primárias de seleção.

A seleção dos setores censitários em cada um dos municípios foi realizada tendo como critério de eliminação os setores com menos de vinte domicílios particulares permanentes.

Em cada setor da amostra, foram selecionados dezesseis domicílios que foram escolhidos de acordo com a cota estabelecida por sexo e faixa etária. Cada estrato da amostra foi submetido ao respectivo fator de expansão, permitindo análise dos resultados por inferência para 185 municípios para o estado de Pernambuco. O nível de confiança adotado foi de 95\%.

A amostra final foi estabelecida em 640 indivíduos com idade igual ou acima de 18 anos. Em cada setor, foram realizadas duas entrevistas adicionais para assegurar número suficiente de crianças com até dois anos de idade para análise estatística. A entrevista foi conduzida com a mãe da criança. O tamanho final da amostra ficou então em 720 indivíduos. Dos 720 indivíduos selecionados aleatoriamente, 591 se encaixavam nos critérios de inclusão do estudo.

$\mathrm{O}$ instrumento utilizado foi adaptado do questionário brasileiro, por ocasião da realização da Pesquisa Mundial de Saúde (PMS). O questionário é composto de sete módulos de perguntas fechadas; para esta análise, foi utilizado o módulo de avaliação da resposta do sistema de saúde.

O trabalho de campo foi realizado por empresa contratada para esta finalidade (Ibope Opinião). No trabalho de campo, foi realizado processo permanente de crítica aos questionários respondidos, visando à consistência das respostas fornecidas.

Inicialmente foi realizada uma análise descritiva com base nas variáveis selecionadas para este estudo, seguida pela descrição do grau de satisfação a partir de um conjunto de variáveis que expressam o grau de satisfação do usuário.

A variável dependente foi "satisfação dos usuários", definida como o percentual de respostas de grau excelente ou bom para todas aquelas em que as opções contemplavam cinco níveis (nível 1 - excelente; a nível 5 - péssimo).

As variáveis independentes foram sexo, idade (estratificada por faixa etária de 18 a 39 anos e 40 anos ou mais), filiação a plano privado de saúde (SUS e não SUS), tipo de assistência recebida (ambulatorial ou hospitalar), variáveis relativas ao 
perfil socioeconômico dos usuários (grau de escolaridade, situação de trabalho e número de bens do domicílio).

A partir da variável número de bens do domicílio foi construído um índice chamado indicador de bens (IB), conforme metodologia empregada por Szwarcwald et al. ${ }^{17}$. Utilizou-se a fórmula IB $=\Sigma_{i}\left(1-f_{i}\right) b_{i}$, em que $i$ varia de 1 até 10 bens, $\mathrm{b}_{i}$ é igual a 1 ou a zero, respectivamente na presença ou ausência de bem. O peso atribuído à presença de cada bem no domicílio foi o complementar da frequência relativa $\left(f_{i}\right)$ de cada bem na amostra total, isto é, quanto mais rara for a presença do bem, maior o peso atribuído a ele.

Os seguintes aspectos foram analisados: habilidades do profissional de saúde; disponibilidade de equipamentos; disponibilidade de medicamentos; resolução do problema; tempo gasto com o deslocamento até o serviço; tempo de espera para o atendimento; tratamento respeitoso por parte dos profissionais de saúde; respeito durante exames físicos; clareza nas explicações dos profissionais; disponibilidade de tempo para fazer perguntas sobre o problema de saúde ou tratamento; possibilidade de obter informações sobre tratamentos ou exames alternativos; participação na tomada de decisões; privacidade junto aos profissionais de saúde; sigilo das informações; liberdade de escolha do profissional de saúde; limpeza das instalações dos serviços; espaço disponível das salas de espera e atendimento.

Os percentuais de satisfação foram analisados por "tipo de atendimento" - ambulatorial ou internação hospitalar - e por "forma de pagamento" - não pagou (SUS), pagamento por desembolso direto ou por meio do plano de saúde (não SUS).

Foi perguntado ainda ao indivíduo se ele se sentiu discriminado no serviço de saúde por algum dos seguintes motivos: sexo, idade, falta de dinheiro, classe social, preferência sexual e tipo de doença. Calculou-se a proporção de pessoas que se sentiram discriminadas por quaisquer motivos ora perguntados.

Na segunda etapa da análise, com o objetivo de explicar a variação total do conjunto de dados através de um pequeno número de fatores, foi utilizada a técnica de análise fatorial, com extração de componentes principais (ACP), que permite transformar linearmente o conjunto de variáveis originais em um conjunto substancialmente menor de variáveis não correlacionadas, as quais representam a maioria da informação contida no conjunto original de variáveis. Após procedimento de rotação para obtenção de uma estrutura simples da matriz de cargas (591 indivíduos x 17 questões), foram identificados os três eixos ou componentes capazes de apontar os diferentes aspectos da satisfação, atribuindo-se um escore para cada indivíduo relativo a cada eixo da matriz. Os escores de cada componente principal foram transformados em uma escala variando de 0 a 100, em que o valor zero corresponde ao escore mínimo de satisfação, enquanto o valor 100, ao nível máximo.

Testes $(\mathrm{t})$ foram realizados para analisar as diferenças no escore médio de satisfação de cada componente segundo as seguintes variáveis: sexo; grau de escolaridade - ensino fundamental incompleto, ensino fundamental completo e mais; faixa etária - 18 a 39 anos, 40 anos e mais; sentimento de discriminação por algum motivo - sim, não; forma de pagamento - SUS, não-SUS; situação de trabalho - trabalha, desempregado; indicador de bens - até 1 e $>1$.

$\mathrm{Na}$ terceira etapa, para cada componente encontrado, foram identificadas as variáveis mais fortemente correlacionadas, sendo realizada análise com modelo de regressão linear múltipla para os dois tipos de atendimento. O escore médio da satisfação foi utilizado para fazer a regressão de cada componente principal. A variável dependente foi o escore de satisfação, e as variáveis independentes foram sexo, escolaridade, idade, discriminação, forma de pagamento, situação de trabalho e indicador de bens.

Para a análise estatística, foi utilizado o programa SPSS (versão 8.0). A análise foi feita no nível individual, tendo como variável resposta o escore de satisfação.

\section{Resultados}

Dos 591 usuários entrevistados, $87,6 \%$ receberam assistência ambulatorial no ano anterior à pesquisa, sendo 73,0\% de usuários do Sistema Único de Saúde (SUS). Em relação aos 12,4\% dos usuários que se internaram no ano anterior à pesquisa, $84,9 \%$ utilizaram o SUS no seu atendimento.

A população-alvo deste estudo foi de pessoas com 18 anos ou mais. Dentre esta população, as pessoas com idade de 18 a 39 anos representam 47,6\% de indivíduos no estado de Pernambuco.

A análise do perfil socioeconômico e demográfico das pessoas que receberam atendimento ambulatorial revelou que, dentre os usuários do SUS, 59,8\% tinham entre 18 e 39 anos de idade, enquanto dentre os que pagaram pelo atendimento, $69,3 \%$ pertenciam a essa faixa. O sexo femini- 
no predominou nas duas formas de atendimento. Pessoas com ensino fundamental completo representavam 69,3\% dos usuários não-SUS, enquanto para os usuários do SUS predominaram aqueles com ensino fundamental incompleto (70,6\%). Mais de 50\% dos usuários, independentemente da forma de pagamento, são pessoas que não trabalham e com pouco número de bens, sendo que para o SUS esses percentuais são superiores aos encontrados para os usuários não-SUS. As diferenças de proporções das variáveis escolaridade, situação de trabalho e número de bens foram significantes, quando comparados os usuários do SUS em relação aos não-SUS.

Das pessoas que se internaram no ano anterior ao período da coleta dos dados, a análise revelou que o SUS foi responsável por $67,8 \%$ das internações na faixa etária de 18 a 39 anos e 32,2\% das pessoas com 40 anos ou mais. Entre os usuários não-SUS, $72,8 \%$ tinham entre 18 e 39 anos de idade e $27,2 \%$ tinham 40 anos ou mais. O sexo feminino foi predominante, independentemente da forma de pagamento da assistência (58,3\% não-SUS e 78,7\% SUS). A grande maioria dos usuários não-SUS tem ensino fundamental completo ou mais $(83,3 \%)$, enquanto dentre os usuários do SUS o predomínio foi de pessoas com o nível de escolaridade do ensino fundamental incompleto $(63,9 \%)$. Como revelado para o atendimento ambulatorial, mais de 50\% dos usuários, independentemente da forma de pagamento, são pessoas que não trabalham e com pouco número de bens, sendo que para o SUS esses percentuais são bem superiores aos encontrados para os usuários não-SUS. A escolaridade foi a única variável que revelou diferenças significantes entre os usuários do SUS em relação aos não-SUS.

Quanto aos aspectos relacionados à avaliação, para as duas formas de atendimento, "a disponibilidade de medicamentos" e "o tempo de espera até o atendimento" obtiveram os menores graus de satisfação por parte dos usuários, dentre todos os aspectos analisados. O "respeito à intimidade" e o "sigilo das informações pessoais" obtiveram os maiores percentuais de satisfação. Soma-se o aspecto "Habilidades do profissional de saúde" ao elevado percentual de satisfação quando da internação hospitalar (Tabela 1).

Ainda analisando a Tabela 1, praticamente todos os aspectos do atendimento ambulatorial foram mais mal avaliados pelos usuários do SUS em relação aos não-SUS. O mesmo não aconteceu quando o indivíduo precisou ser internado. O "tempo de deslocamento" e a "participação nas decisões sobre a conduta” foram mais bem ava- liados pelos usuários do SUS em relação aos não-SUS.

Cerca de $1 \%$ dos usuários que receberam atendimento ambulatorial pelo SUS se sentiram discriminados por falta de dinheiro e pela classe social. Em relação à análise da discriminação quando o usuário foi internado, cerca de 1,5\% dos usuários (SUS) se sentiram discriminados pelos mesmos motivos que os usuários do atendimento ambulatorial, e $8,3 \%$ (não-SUS) se sentiram discriminados em razão da cor da pele. Salienta-se que, pelo tamanho da amostra, nenhuma diferença entre SUS e não-SUS mostrouse estatisticamente significativa.

A Tabela 2 revela os resultados da análise segundo os componentes principais, mostrando que para o atendimento ambulatorial, $24,3 \%$ da variação foi explicada pelo primeiro componente, que está relacionado com os fatores ligados ao profissional de saúde. O segundo componente explica $19,3 \%$ da variação total e está relacionado aos aspectos ligados aos serviços de saúde, e o último componente explica $11,2 \%$ e está relacionado à resolutividade do problema de saúde.

Para a internação hospitalar, $33,8 \%$ da variação foi explicada pelo primeiro componente, seguindo-se $15,7 \%$ para o segundo e $14,5 \%$ para o terceiro.

O ponto de corte para análise de correlação das variáveis do estudo em relação a cada componente encontrado foi de $50 \%$ (0,5 em valor absoluto) e está em negrito.

Para os dois tipos de atendimento analisados neste estudo, as variáveis de avaliação sobre clareza das explicações do profissional de saúde, tempo para fazer perguntas sobre problema ou tratamento de saúde, possibilidade de obter informações sobre tratamentos alternativos, privacidade com os profissionais de saúde e sigilo das informações pessoais obtiveram valores de correlação maiores no componente 1 , que está relacionado aos fatores ligados ao profissional de saúde.

Para o atendimento ambulatorial somam-se mais duas variáveis fortemente correlacionadas ao componente 1 , as quais foram a participação nas decisões sobre a conduta e a liberdade de escolha do profissional de saúde.

Já para o atendimento hospitalar, foram observadas mais três variáveis correlacionadas ao componente ligado ao profissional de saúde: habilidades do profissional de saúde, respeito na maneira de receber e tratar o usuário e respeito à intimidade durante exame físico.

O segundo componente analisado está relacionado aos aspectos ligados aos serviços de saúde, 
e para o atendimento ambulatorial as variáveis mais fortemente correlacionadas foram a disponibilidade de equipamentos, a disponibilidade de medicamentos, o tempo de espera até ser atendido, a limpeza das instalações incluindo banheiros e o espaço das salas de espera e atendimento.

Quando se analisou o componente 2, segundo o atendimento por internação hospitalar, o tempo de deslocamento e a participação nas decisões sobre a conduta foram as variáveis que tiveram maior correlação.

O último componente analisado está ligado à resolutividade do problema de saúde, e os respondentes que tiveram atendimento ambulatorial avaliaram a resolução de seu problema, o tempo de deslocamento e o respeito à intimidade

Tabela 1. Percentual (\%) de satisfação segundo tipo de assistência, forma de pagamento e aspectos relacionados à avaliação do atendimento. Pernambuco, 2005.

\begin{tabular}{|c|c|c|c|c|c|c|}
\hline & \multicolumn{4}{|c|}{ Forma de pagamento } & & \\
\hline & \multicolumn{2}{|c|}{ Não-SUS } & \multicolumn{2}{|c|}{ SUS } & \multicolumn{2}{|c|}{ Total } \\
\hline & $\mathrm{N}$ & $\%$ & $\mathrm{~N}$ & $\%$ & $\mathrm{~N}$ & $\%$ \\
\hline \multicolumn{7}{|l|}{ Atendimento ambulatorial - Aspecto } \\
\hline Resolução do problema & 121 & 87,7 & 287 & 77,2 & 408 & 80,0 \\
\hline Tempo de deslocamento & 102 & 72,9 & 271 & 72,3 & 373 & 72,4 \\
\hline Tempo de espera até ser atendido & 96 & 68,6 & 217 & 57,9 & 313 & 60,8 \\
\hline Respeito no tratamento & 133 & 95,0 & 317 & 84,3 & 450 & 87,2 \\
\hline Respeito à intimidade & 133 & 96,4 & 335 & 90,8 & 468 & 92,3 \\
\hline Clareza das explicações & 134 & 95,7 & 328 & 87,2 & 462 & 89,5 \\
\hline Tempo para fazer perguntas & 128 & 91,4 & 310 & 82,4 & 438 & 84,9 \\
\hline $\begin{array}{l}\text { Possibilidade obter informações sobre tratamentos } \\
\text { alternativos }\end{array}$ & 116 & 85,3 & 283 & 77,1 & 399 & 79,3 \\
\hline Participação nas decisões sobre a conduta & 122 & 87,8 & 294 & 79,9 & 416 & 82,1 \\
\hline Privacidade com os profissionais de saúde & 131 & 94,2 & 324 & 86,6 & 455 & 88,7 \\
\hline Sigilo das informações pessoais & 132 & 95,7 & 332 & 89,7 & 464 & 91,3 \\
\hline Liberdade de escolha do profissional de saúde & 120 & 87,0 & 266 & 73,7 & 386 & 77,4 \\
\hline Limpeza das instalações & 127 & 94,1 & 264 & 73,1 & 391 & 78,8 \\
\hline Espaço das salas de espera e dos exames & 122 & 87,1 & 274 & 73,3 & 396 & 77,0 \\
\hline Habilidades do profissional de saúde satisfatórias & 134 & 96,4 & 308 & 81,9 & 442 & 85,8 \\
\hline Disponibilidade de equipamentos & 123 & 87,9 & 269 & 71,7 & 392 & 76,1 \\
\hline Disponibilidade de medicamentos & 63 & 56,3 & 205 & 56,6 & 268 & 56,5 \\
\hline \multicolumn{7}{|l|}{ Internação hospitalar - Aspecto } \\
\hline Resolução do problema & 11 & 91,7 & 46 & 75,4 & 57 & 78,1 \\
\hline Tempo de deslocamento & 7 & 58,3 & 46 & 78,0 & 53 & 74,6 \\
\hline Tempo de espera até ser atendido & 8 & 66,7 & 38 & 64,4 & 46 & 64,8 \\
\hline Respeito no tratamento & 11 & 91,7 & 52 & 83,9 & 63 & 85,1 \\
\hline Respeito à intimidade & 12 & 100,0 & 54 & 87,1 & 66 & 89,2 \\
\hline Clareza das explicações & 12 & 100,0 & 51 & 83,6 & 63 & 86,3 \\
\hline Tempo para fazer perguntas & 11 & 100,0 & 48 & 78,7 & 59 & 81,9 \\
\hline $\begin{array}{l}\text { Possibilidade obter informações sobre tratamentos } \\
\text { alternativos }\end{array}$ & 11 & 100,0 & 44 & 72,1 & 55 & 76,4 \\
\hline Participação nas decisões sobre a conduta & 10 & 83,3 & 51 & 85,0 & 61 & 84,7 \\
\hline Privacidade com os profissionais de saúde & 11 & 100,0 & 48 & 78,7 & 59 & 81,9 \\
\hline Sigilo das informações pessoais & 12 & 100,0 & 53 & 86,9 & 65 & 89,0 \\
\hline Liberdade de escolha do profissional de saúde & 11 & 100,0 & 43 & 71,7 & 54 & 76,1 \\
\hline Limpeza das instalações & 12 & 100,0 & 35 & 59,3 & 47 & 66,2 \\
\hline Espaço das salas de espera e dos exames & 9 & 81,8 & 39 & 66,1 & 48 & 68,6 \\
\hline Habilidades do profissional de saúde satisfatórias & 12 & 100,0 & 55 & 88,7 & 67 & 90,5 \\
\hline Disponibilidade de equipamentos & 11 & 100,0 & 51 & 83,6 & 62 & 86,1 \\
\hline Disponibilidade de medicamentos & 8 & 67,8 & 35 & 58,3 & 43 & 59,7 \\
\hline
\end{tabular}

Fonte: Pesquisa Mundial de Saúde-AB, Pernambuco, 2005. 
durante exame físico como sendo as variáveis mais fortemente correlacionadas ao componente 3. A análise desse componente, quando da internação hospitalar, apontou a disponibilidade de equipamentos, a limpeza das instalações incluindo banheiros e o espaço das salas de espera e atendimento como sendo as variáveis com maior valor de carga.
Ainda analisando a Tabela 2, para o atendimento ambulatorial, a variável "habilidades do profissional de saúde" está relacionada aos componentes ligados ao profissional de saúde e ao serviço de saúde. A variável "respeito na maneira de recebê-lo e tratá-lo” está correlacionada aos três componentes analisados, ou seja, para o usuário, o respeito na maneira de ser recebido e tratado está

Tabela 2. Resultados da análise fatorial por tipo de assistência: cargas nos três primeiros componentes principais. Pernambuco, 2005.

Respostas satisfatórias (avaliadas como excelente e bom).

\begin{tabular}{|c|c|c|c|}
\hline & \multicolumn{3}{|c|}{ Componentes } \\
\hline & 1 & 2 & 3 \\
\hline \multicolumn{4}{|l|}{ Atendimento ambulatorial - Aspecto (Avaliação) } \\
\hline Habilidades do profissional de saúde & 0,476 & 0,479 & 0,121 \\
\hline Disponibilidade de equipamentos & 0,416 & 0,644 & 0,018 \\
\hline Disponibilidade de medicamentos & 0,148 & 0,618 & 0,247 \\
\hline Resolução do seu problema & 0,209 & 0,271 & 0,576 \\
\hline Tempo de deslocamento & 0,028 & 0,085 & 0,731 \\
\hline Tempo de espera até ser atendido & 0,100 & 0,557 & 0,390 \\
\hline Respeito na maneira de recebê-lo e tratá-lo & 0,466 & 0,380 & 0,400 \\
\hline Respeito à intimidade durante exame físico & 0,366 & 0,043 & 0,577 \\
\hline Clareza das explicações do profissional de saúde & 0,704 & 0,229 & 0,119 \\
\hline Tempo para fazer perguntas sobre problema ou tratamento & 0,713 & 0,289 & 0,008 \\
\hline Possibilidade de obter informações sobre tratamentos alternativos & 0,663 & 0,437 & 0,038 \\
\hline Participação nas decisões sobre a conduta & 0,761 & 0,087 & 0,222 \\
\hline Privacidade com os profissionais de saúde & 0,670 & 0,114 & 0,317 \\
\hline Sigilo das informações pessoais & 0,720 & 0,038 & 0,364 \\
\hline Liberdade de escolha do profissional de saúde & 0,516 & 0,408 & 0,077 \\
\hline Limpeza das instalações, incluindo banheiros & 0,206 & 0,752 & 0,066 \\
\hline Espaço das salas de espera e atendimento & 0,093 & 0,803 & 0,083 \\
\hline$\%$ de variância & $24,3 \%$ & $19,3 \%$ & $11,2 \%$ \\
\hline \multicolumn{4}{|l|}{ Internação hospitalar - Aspecto (avaliação) } \\
\hline Habilidades do profissional de saúde & 0,868 & 0,049 & 0,111 \\
\hline Disponibilidade de equipamentos & 0,339 & 0,433 & 0,547 \\
\hline Disponibilidade medicamentos & 0,234 & 0,303 & 0,479 \\
\hline Resolução do seu problema & 0,593 & 0,345 & 0,262 \\
\hline Tempo de deslocamento & $-0,084$ & 0,864 & 0,060 \\
\hline Tempo de espera até ser atendido & 0,396 & 0,379 & $-0,010$ \\
\hline Respeito na maneira de recebê-lo e tratá-lo & 0,721 & $-0,147$ & 0,235 \\
\hline Respeito à intimidade durante exame físico & 0,882 & 0,034 & 0,155 \\
\hline Clareza das explicações do profissional de saúde & 0,740 & 0,305 & 0,149 \\
\hline Tempo para fazer perguntas sobre problema ou tratamento & 0,760 & 0,379 & 0,023 \\
\hline Possibilidade de obter informações sobre tratamentos alternativos & 0,607 & 0,452 & 0,107 \\
\hline Participação nas decisões sobre a conduta & 0,387 & 0,654 & 0,048 \\
\hline Privacidade com os profissionais de saúde & 0,749 & 0,374 & 0,032 \\
\hline Sigilo das informações pessoais & 0,787 & 0,339 & 0,184 \\
\hline Liberdade de escolha do profissional de saúde & 0,428 & 0,486 & 0,303 \\
\hline Limpeza das instalações, incluindo banheiros & 0,056 & 0,023 & 0,896 \\
\hline Espaço das salas de espera e atendimento & 0,061 & $-0,063$ & 0,900 \\
\hline$\%$ de variância & $33,8 \%$ & $15,7 \%$ & $14,5 \%$ \\
\hline
\end{tabular}

Fonte: Pesquisa Mundial de Saúde-AB, Pernambuco, 2005. 
relacionado ao profissional de saúde, ao serviço de saúde e à resolutividade do problema de saúde.

A disponibilidade de medicamentos e a liberdade de escolha do profissional de saúde são variáveis que estão correlacionadas aos três componentes analisados, quando se avalia a assistência hospitalar. A variável "tempo de espera até ser atendido" transita ora nos componentes ligados ao profissional de saúde, ora no componente ligado ao serviço de saúde.

A análise das diferenças nos escores médios de satisfação está apresentada na Tabela 3. Em relação ao atendimento ambulatorial, o componente relativo às condições dos serviços de saúde apresentou o menor grau médio de satisfação $(59,31)$ na ótica do usuário. O terceiro componente, relativo à resolutividade do problema de saúde, obteve um grau médio de satisfação $(62,61)$ melhor do que o componente anterior, mas não se distanciou muito da avaliação relativa aos serviços de saúde. O componente que apresentou o maior grau médio de satisfação foi o relativo à avaliação dos profissionais de saúde, com valor próximo a 70.

Quando a assistência se deu através de internação hospitalar, o componente que apresentou maior grau médio de satisfação foi o relativo à avaliação do profissional de saúde $(72,36)$, distanciando-se, relevantemente, do componente ligado às condições dos serviços de saúde $(64,74)$ e do componente que mede a satisfação em relação ao componente referente à resolução dos problemas de saúde $(62,05)$ (Tabela 3 ).

Para o atendimento ambulatorial, os graus médios de satisfação mostram diferenças significativas para a escolaridade quando esta se refere aos componentes relacionados ao serviço de saúde e resolução de problemas. As diferenças foram significativas para as variáveis indicador de bens, idade e forma de pagamento em relação aos dois primeiros componentes, sendo estes relativos à avaliação do tratamento recebido dos profissionais de saúde e das condições dos serviços de saúde (respectivamente). Houve diferença significante para a variável discriminação quando esta se deu em relação ao tratamento recebido por parte dos profissionais de saúde para os dois tipos de atendimento recebido (Tabela 3).

Ainda na Tabela 3, a análise das diferenças dos escores médios de satisfação para o atendimento hospitalar revelou que existem diferenças significantes nos escores médios de satisfação para as variáveis sexo e situação de trabalho, quando esta está relacionada às condições dos serviços de saúde. As variáveis idade e forma de pagamento apresentaram diferenças significantes ape- nas quando estas estão relacionadas à resolutividade dos problemas de saúde.

A análise da influência conjunta das variáveis sexo, idade, grau de escolaridade, situação de trabalho, indicador de bens, forma de pagamento e sentimento de discriminação sobre o grau de satisfação com o atendimento, por componente, revelou que apenas o sentimento de discriminação foi consistentemente associado ao grau de satisfação com o atendimento na assistência ambulatorial. Embora os coeficientes correspondentes às demais variáveis tenham sido significativos em alguns componentes, os efeitos não foram sistemáticos e algumas vezes se mostraram com sentidos contrários (Tabela 4).

Em síntese, para o atendimento ambulatorial, independentemente do número de bens que o usuário possua, ser jovem, ter sofrido alguma discriminação e ser usuário do SUS gerou menores graus de satisfação em relação aos componentes que apresentam os fatores relacionados ao profissional de saúde e às condições dos serviços de saúde. Curiosamente, ter baixa escolaridade não influenciou num menor grau de satisfação quando se analisou o componente relacionado às condições dos serviços de saúde.

Nesse mesmo tipo de atendimento, ser mulher e ter sofrido alguma discriminação está fortemente associado a um menor grau de satisfação quando da resolução do problema de saúde.

Quando da internação hospitalar, ter sido discriminado e ser usuário do SUS geraram menor satisfação com os fatores relacionados ao profissional de saúde. Para o componente ligado às condições dos serviços de saúde, ser jovem e desempregado resultou em menor satisfação, enquanto ser mulher e ter maior número de bens apresentou melhores graus de satisfação para este componente.

Os jovens e usuários do SUS, invariavelmente, sentiram-se menos satisfeitos com a resolução do problema de saúde quando precisaram ser internados.

\section{Discussão}

A avaliação da satisfação na ótica dos usuários dos serviços de saúde exibe quadro complexo, ainda em fase de produção de conhecimento mais do que de consolidação desse conhecimento, exigindo dos pesquisadores grande flexibilidade na escolha do método avaliativo e dos indicadores que mais se ajustem às suas realidades e às necessidades do sistema. 
Tabela 3. Média e erro padrão dos escores de satisfação por componente principal e tipo de assistência segundo variáveis sociodemográficas, forma de pagamento e sentimento de discriminação.

Pernambuco, 2005.

\begin{tabular}{|c|c|c|c|}
\hline & \multicolumn{3}{|c|}{ Componentes } \\
\hline & $\begin{array}{l}\text { Profissional de } \\
\text { saúde }\end{array}$ & $\begin{array}{l}\text { Serviço de } \\
\text { saúde }\end{array}$ & $\begin{array}{l}\text { Resolução de } \\
\text { problemas }\end{array}$ \\
\hline \multicolumn{4}{|c|}{ Atendimento ambulatorial - Variáveis } \\
\hline \multicolumn{4}{|c|}{ Sexo } \\
\hline Masculino & $69,83 \pm 1,42$ & $59,93 \pm 1,78$ & $64,63 \pm 1,18$ \\
\hline Feminino & $70,54 \pm 1,06$ & $58,97 \pm 1,24$ & $61,52 \pm 0,95$ \\
\hline \multicolumn{4}{|l|}{ Escolaridade } \\
\hline Fundamental incompleto & $70,18 \pm 1,06$ & $61,69 \pm 1,17$ & $61,54 \pm 1,00$ \\
\hline Fundamental completo e + & $70,47 \pm 1,43$ & $55,46 \pm 1,85^{\star *}$ & $64,36 \pm 1,08^{*}$ \\
\hline \multicolumn{4}{|l|}{ Situação de trabalho } \\
\hline Trabalha & $71,09 \pm 1,31$ & $60,03 \pm 1,68$ & $62,68 \pm 1,27$ \\
\hline Desempregado & $69,80 \pm 1,11$ & $58,87 \pm 1,28$ & $62,58 \pm 0,92$ \\
\hline \multicolumn{4}{|l|}{ Indicador de bens } \\
\hline Até 1 & $71,92 \pm 1,15$ & $62,38 \pm 1,37$ & $61,59 \pm 1,16$ \\
\hline$>1$ & $69,00 \pm 1,21^{* *}$ & $56,90 \pm 1,45^{\star *}$ & $63,42 \pm 0,98$ \\
\hline \multicolumn{4}{|l|}{ Idade } \\
\hline 18 a 39 anos & $68,74 \pm 1,14$ & $56,92 \pm 1,36$ & $62,35 \pm 0,94$ \\
\hline 40 anos e + & $73,17 \pm 1,14^{\star *}$ & $63,74 \pm 1,39^{* *}$ & $63,11 \pm 1,23$ \\
\hline \multicolumn{4}{|l|}{ Discriminação } \\
\hline Sim & $41,18 \pm 8,55$ & $37,47 \pm 5,69$ & $51,28 \pm 6,79$ \\
\hline Não & $70,90 \pm 0,82^{*}$ & $59,77 \pm 1,02$ & $62,85 \pm 0,75$ \\
\hline \multicolumn{4}{|l|}{ Forma de pagamento } \\
\hline Não SUS & $73,27 \pm 1,37$ & $66,14 \pm 1,44$ & $63,15 \pm 1,28$ \\
\hline SUS & $69,28 \pm 1,03^{*}$ & $56,99 \pm 1,25^{\star *}$ & $62,43 \pm 0,90$ \\
\hline Total & $70,29 \pm 0,85$ & $59,31 \pm 1,02$ & $62,61 \pm 0,75$ \\
\hline \multicolumn{4}{|c|}{ Internação hospitalar - Variáveis } \\
\hline \multicolumn{4}{|c|}{ Sexo } \\
\hline Masculino & $72,21 \pm 4,84$ & $58,78 \pm 6,95$ & $63,42 \pm 7,19$ \\
\hline Feminino & $73,67 \pm 3,00$ & $66,36 \pm 2,57^{\star}$ & $61,67 \pm 3,89$ \\
\hline \multicolumn{4}{|l|}{ Escolaridade } \\
\hline Fundamental incompleto & $70,74 \pm 3,71$ & $62,35 \pm 3,61$ & $68,91 \pm 4,19$ \\
\hline Fundamental completo e + & $76,40 \pm 3,45$ & $67,52 \pm 3,43$ & $54,09 \pm 5,23$ \\
\hline \multicolumn{4}{|l|}{ Situação de trabalho } \\
\hline Trabalha & $77,86 \pm 3,66$ & $72,42 \pm 3,12$ & $5,75 \pm 5,98$ \\
\hline Desempregado & $71,64 \pm 3,24$ & $61,82 \pm 3,17^{\star}$ & $66,34 \pm 3,97$ \\
\hline \multicolumn{4}{|l|}{ Indicador de bens } \\
\hline Até 1 & $74,38 \pm 3,50$ & $67,41 \pm 3,54$ & $64,64 \pm 5,02$ \\
\hline$>1$ & $72,53 \pm 3,70$ & $62,60 \pm 3,52$ & $59,96 \pm 4,65$ \\
\hline \multicolumn{4}{|l|}{ Idade } \\
\hline 18 a 39 anos & $75,90 \pm 2,85$ & $63,96 \pm 3,30$ & $55,34 \pm 4,43$ \\
\hline 40 anos e + & $67,98 \pm 5,13$ & $66,40 \pm 3,59$ & $76,21 \pm 3,42^{* *}$ \\
\hline \multicolumn{4}{|l|}{ Discriminação } \\
\hline Sim & $31,62 \pm 37,94$ & $59,29 \pm 9,31$ & $82,37 \pm 11,10$ \\
\hline Não & $74,70 \pm 2,30^{\star *}$ & $64,92 \pm 2,58$ & $61,39 \pm 3,47$ \\
\hline \multicolumn{4}{|l|}{ Forma de pagamento } \\
\hline Não SUS & $82,51 \pm 2,45$ & $62,41 \pm 5,49$ & $74,83 \pm 4,72$ \\
\hline SUS & $71,56 \pm 2,97$ & $65,20 \pm 2,82$ & $59,54 \pm 3,89^{*}$ \\
\hline Total & $72,36 \pm 2,56$ & $64,74 \pm 2,51$ & $62,05 \pm 3,40$ \\
\hline
\end{tabular}

Fonte: Pesquisa Mundial de Saúde-AB, Pernambuco, 2005.

"Significante ao nível de 5\%; " Significante ao nível de $1 \%$. 
Tabela 4. Resultados dos modelos de regressão linear múltipla tendo como variáveis dependentes os escores de satisfação por componente principal e tipo de assistência. Pernambuco, 2005.

\begin{tabular}{|c|c|c|c|c|c|c|}
\hline & \multicolumn{2}{|c|}{$\begin{array}{l}\text { Profissional } \\
\text { de saúde }\end{array}$} & \multicolumn{2}{|c|}{$\begin{array}{l}\text { Serviço } \\
\text { de saúde }\end{array}$} & \multicolumn{2}{|c|}{$\begin{array}{c}\text { Resolução } \\
\text { de problemas }\end{array}$} \\
\hline & $\beta$ & Valor de $\mathrm{p}$ & $\beta$ & Valor de $\mathrm{p}$ & $\beta$ & Valor de $\mathrm{p}$ \\
\hline \multicolumn{7}{|c|}{ Atendimento ambulatorial - Variáveis } \\
\hline Sexo feminino & 0,54 & 0,767 & $-0,85$ & 0,689 & $-3,68$ & 0,026 \\
\hline Fundamental incompleto & $-1,37$ & 0,483 & 7,47 & 0,001 & $-3,37$ & 0,057 \\
\hline Desempregado & $-1,11$ & 0,537 & $-1,24$ & 0,554 & 1,57 & 0,334 \\
\hline Indicador de bens & 3,43 & 0,055 & 5,63 & 0,007 & $-1,16$ & 0,471 \\
\hline Idade (18-39 anos) & $-4,40$ & 0,015 & $-5,13$ & 0,015 & $-1,27$ & 0,434 \\
\hline Foi discriminado & $-26,35$ & $<0,001$ & $-15,16$ & 0,029 & $-13,11$ & 0,014 \\
\hline SUS & $-3,95$ & 0,056 & $-13,58$ & $<0,001$ & 1,33 & 0,474 \\
\hline \multicolumn{7}{|c|}{ Internacão hospitalar - Variáveis } \\
\hline Sexo feminino & 6,87 & 0,325 & 17,03 & 0,016 & 15,94 & 0,074 \\
\hline Fundamental incompleto & $-2,53$ & 0,680 & $-9,69$ & 0,116 & 12,42 & 0,114 \\
\hline Desempregado & $-5,25$ & 0,370 & $-13,90$ & 0,019 & 7,10 & 0,339 \\
\hline Indicador de bens & 8,08 & 0,152 & 13,92 & 0,015 & 7,37 & 0,301 \\
\hline Idade (18-39 anos) & 1,66 & 0,793 & $-16,28$ & 0,012 & $-21,62$ & 0,009 \\
\hline Foi discriminado & $-49,79$ & 0,001 & $-16,08$ & 0,252 & 11,3 & 0,536 \\
\hline SUS & $-14,92$ & 0,045 & $-0,22$ & 0,975 & $-29,60$ & 0,002 \\
\hline
\end{tabular}

Fonte: Pesquisa Mundial de Saúde-AB, Pernambuco, 2005.

A análise dos dados da PMS-AB revelou que, no sistema de saúde pernambucano, existe predominância de atendimentos às pessoas jovens, do sexo feminino, em sua maioria sem trabalho e com poucos bens por domicílio. Os níveis de escolaridade mais baixos foram predominantes entre os usuários do SUS em relação às pessoas que de alguma forma pagaram pelo seu atendimento. Segundo Bós e Bós ${ }^{18}$, pessoas com melhor educação teriam melhor conhecimento sobre a sua situação de saúde e, em geral, melhores condições socioeconômicas, o que possibilitaria a procura de outra alternativa de atendimento incluindo aqueles pagos. Os resultados do presente trabalho confirmam essa hipótese.

Conforme explicam Souza e Pereira ${ }^{19}$, a ideia que o paciente tem de saúde irá influenciar no julgamento da qualidade. Alguns autores ${ }^{20,21} \mathrm{di}$ zem que, na avaliação da qualidade dos serviços de saúde, sobretudo no que diz respeito à satisfação, devemos conhecer as necessidades e os desejos dos pacientes.

Neste trabalho, o tempo de espera até o atendimento e a disponibilidade de medicamentos gerou os menores percentuais de satisfação dos usuários do sistema de saúde em Pernambuco, o que leva a refletir se o acesso está de fato relacionado à oferta de serviços adequados às necessi- dades da população ${ }^{22}$ ou não, e à política de assistência farmacêutica no estado. Esses resultados corroboram os achados de outros autores ${ }^{23,24}$, que revelam, entre os aspectos mais negativos do atendimento nas unidades de saúde, a demora no atendimento e a falta de medicamentos.

Para Ferri et al. ${ }^{14}$, em estudo realizado em uma cidade do interior de São Paulo, a demora para o atendimento foi motivo de grande insatisfação por parte de quem usa os serviços de saúde. Segundo essas autoras, como o atendimento oferecido foi considerado bom, os usuários se satisfizeram pela resolubilidade. As autoras afirmam que o tempo de espera deve ser uma preocupação por parte da equipe no que diz respeito ao atendimento, pois está muito longe de se ter qualidade nesse quesito.

Com o processo de descentralização da assistência farmacêutica, promovendo a gestão local dos recursos financeiros e a operacionalização da aquisição de medicamentos, houve um avanço na direção de um dos eixos que estruturam a organização do SUS, mas evidenciaram-se limites e dificuldades concernentes ao seu processo de implantação. O medicamento é um insumo estratégico de suporte às ações de saúde, cuja falta pode significar interrupções constantes no tratamento, o que afeta a qualidade de vida 
dos usuários e gera insatisfação com o sistema de saúde como um todo ${ }^{14,25}$.

Desde que a Política Nacional de Medicamentos foi aprovada em 2001, foi garantido à população o acesso aos medicamentos considerados essenciais $^{26}$. Esse preceito vai de encontro aos achados no presente trabalho, pois um dos fatores que mais geraram insatisfação foi a indisponibilidade de medicamentos.

Em relação às dificuldades de acesso, alguns autores $^{27}$ que estudaram a satisfação de beneficiários de planos de saúde dizem que as dificuldades de acesso ao atendimento do serviço público de saúde levam uma parcela grande dos cidadãos a procurar os planos de saúde.

Além dessa dificuldade enfrentada por parcelas significativas da população, somam-se as desigualdades relacionadas ao tempo de espera para os usuários serem atendidos. Pessoto et al. ${ }^{28}$, em pesquisa realizada no estado de São Paulo, demonstrou que os usuários do SUS esperam pelo atendimento quase três vezes mais dos que os usuários que pagam pelos serviços de saúde. Segundo esses autores, é nos serviços ambulatoriais que se encontram as maiores desigualdades desse aspecto, o que corrobora os achados encontrados para este estudo, em que os níveis de satisfação com o tempo de espera para o atendimento são sempre menores para os usuários do SUS.

Por sua vez, os níveis de satisfação em relação aos aspectos associados à relação interpessoal do atendimento foram elevados, o que corrobora os resultados encontrados em um estudo realizado no Sri-Lanka ${ }^{5}$, em que os autores encontraram que em alguns hospitais o nível de satisfação com o atendimento era elevado e estava diretamente relacionado à satisfação com o atendimento médico. Para esses autores, mesmo que o estabelecimento de saúde seja precário em termos de equipamentos, deve-se procurar manter o bom relacionamento interpessoal.

Outro aspecto analisado neste trabalho foi o relativo à discriminação sofrida pelos usuários, sendo encontrados baixos percentuais de indivíduos que relataram ter sofrido algum tipo de discriminação quando de seu atendimento em saúde. Nos dias atuais, com a publicação da Carta dos Direitos dos Usuários da Saúde ${ }^{29}$, torna-se inaceitável que ainda assim persistam, mesmo que em pequeno número, relatos de discriminação na assistência em saúde no estado de Pernambuco.

Na PMS brasileira, os achados revelaram um alto percentual de pessoas que se sentiram discriminadas, A falta de dinheiro e a classe social foram relatadas como as principais causas de dis- criminação $\mathrm{o}^{30}$. Em Pernambuco, o principal motivo relatado foi em relação à cor da pele (8,3\%). Isso remete à reflexão de que, no Brasil, país fortemente inter-racial, ainda persistem aspectos cruciais em relação à discriminação racial.

Através do uso da técnica de componentes principais, foi possível apontar para três grandes eixos, como revelado por Gouveia et al. ${ }^{30}$ na análise da PMS brasileira. Esses eixos estão fortemente correlacionados às variáveis que expressam satisfação dos usuários com o atendimento recebido por parte dos profissionais de saúde, às condições dos serviços de saúde e à resolução dos problemas de saúde.

Os dados da PMS-AB mostraram que o acesso está fortemente correlacionado às condições de atendimento dos serviços de saúde, e esse componente foi o responsável pelo menor grau médio de satisfação encontrado no presente trabalho, como demonstrado por alguns autores ${ }^{30-32}$.

Dentre as variáveis estudadas, as diferenças dos escores médios de satisfação não foram significativas apenas para o sexo e a situação de trabalho, quando do atendimento ambulatorial; já para a internação hospitalar, as diferenças não foram significantes apenas para a escolaridade e para o indicador de bens. Esses resultados revelam que para o estado de Pernambuco, independentemente da forma de pagamento do serviço de saúde, essas variáveis não geram maior ou menor satisfação. Para Travassos et al. ${ }^{33}$, a diminuição da associação da escolaridade com acesso aos serviços de saúde pode estar associada ao aumento do nível de escolaridade da população ocorrido nos últimos anos.

Uma possível limitação deste estudo é inerente ao fato de que o olhar do usuário varia de acordo com a expectativa dele em relação ao atendimento recebido. As manifestações certamente não foram uniformes nem constantes, mas trazem consigo fenômenos sociais, expectativas individuais e coletivas, fatores econômicos, políticos, culturais ${ }^{14}$, que certamente influenciaram os resultados obtidos no estudo.

Concluindo, a partir dos resultados apresentados, foi possível obter um panorama sobre a forma como o sistema de saúde pernambucano tem respondido às expectativas e aos anseios de sua população. É importante enfatizar a necessidade de pesquisas futuras no sentido de aprofundar questões relacionadas à assistência farmacêutica no estado de Pernambuco, bem como as questões relativas à organização dos serviços no intuito de minimizar o tempo de espera para atendimento de seus usuários. 


\section{Colaboradores}

GC Gouveia e WV Souza elaboraram o artigo; CF Luna e PRB Souza Júnior auxiliaram nas análises estatísticas; CL Szwarcwald participou na revisão.

\section{Referências}

1. Szwarcwald CL, Mendonça MHM, Andrade CL. Indicadores de atenção básica em quatro municípios do estado do Rio de Janeiro, 2005: resultados de inquérito domiciliar de base populacional. Cien Saude Colet 2006; 11(3):643-655.

2. Pisco LA. A avaliação como instrumento de mudança. Cien Saude Colet 2006; 11(3):566-568.

3. Malik AM, Schiesari LMC. Qualidade na gestão local de serviços e ações de saúde. São Paulo: Faculdade de Saúde Pública da Universidade de São Paulo; 1998. (Série Saúde e Cidadania, v. 3).

4. Donabedian A. The seven pillars of quality. Arch Pathol Lab Méd 1990; 114:1115-1118.

5. Senarath U, Fernando DN, Rodrigo I. Factors determining client satisfaction with hospital-based perinatal care in Sri Lanka. Tropical Medicine and International Health 2006; 11(9):1442-1451.

6. Murray CJL, Kawabata K, Valentine N. People's experience versus people's expectations: satisfaction measures are profoundly influenced by expectations, say these WHO researchers. Health Affairs 2001; 20(3):21-24.

7. Blendon RJ, Schoen C, Desroches CM, Osborn R, Scoles KL, Zapert K. Inequities in health care: a five-country survey. Access-to-care experiences across the five countries tend to vary along with the countries' insurance coverage policies. Health Affairs 2002; 21(3):182-191.

8. World Health Organization (WHO). Health System Performance: what is the WHO Health System Performance framework? [site da Internet]. [acessado 2008 jun 12]. Disponível em: http://www.who.int/ health-systems-performance/concepts.htm

9. Travassos C, Martins M. Uma revisão sobre os conceitos de acesso e utilização de serviços de saúde. Cad Saude Publica 2004; 20(Supl.2):190-198.

10. Trad LAB, Bastos ACS. O impacto sociocultural do Programa de Saúde da Família (PSF): uma proposta de avaliação. Cad Saude Publica 1998; 14(2):429-435.

11. Esperidião M, Trad LAB. Avaliação de satisfação de usuários. Cien Saude Colet 2005; 10(Supl.):303-312.

12. Stenzel ACB, Misoczky MCA, Oliveira AI. Satisfação dos usuários de serviços públicos de saúde. In: Misoczky MC, Bordin R, organizadores. Gestão local em saúde: práticas e reflexões. Porto Alegre: Dacasa Editora; 2004. p. 87-102.

13. Vaitsman J, Andrade GRB, Farias LO, Médici ME, Nascimento LR. Projeto Gestão de Qualidade e Satisfação dos Usuários em Organizações Públicas de Saúde: um estudo piloto no Instituto de Pesquisa Evandro Chagas-IPEC. Rio de Janeiro: Faperj/Ensp-Fiocruz; 2003. 
14. Ferri SMN, Pereira MJB, Mishima SM, Caccia-Bava MCG, Almeida MCP. As tecnologias leves como geradoras de satisfação em usuários de uma unidade de saúde da família. Interface - Comunic, Saúde, Educ 2007; 11(23):515-529.

15. Ramos DD, Lima MADS. Acesso e acolhimento aos usuários em uma unidade de saúde de Porto Alegre, Rio Grande do Sul, Brasil. Cad Saude Publica 2003; 19(1):27-34.

16. Szwarcwald CL, Viacava F. Pesquisa Mundial de Saúde no Brasil, 2003. Cad Saude Publica 2005; 21(Supl.1):43-53.

17. Szwarcwald CL, Leal MC, Gouveia GC, Souza WV. Desigualdades socioeconômicas em saúde no Brasil: resultados da Pesquisa Mundial de Saúde, 2003. Rev Bras Saúde Matern Infant 2005; 5(Supl.1):S11S22.

18. Bós AMG, Bós AJG. Determinantes na escolha entre atendimento de saúde privada e pública por idosos. Rev Saude Publica 2004; 38(1):113-120.

19. Souza EM, Pereira M. A satisfação do usuário na avaliação dos serviços de saúde. Brasília Médica 1999; 36(1/2):33-36.

20. Tanaka OY. Caminhos alternativos para a institucionalização da avaliação em saúde. Cien Saude Colet 2006; 11(3):571-572.

21. Santos SR, Lacerda MCN. Fatores de satisfação e insatisfação entre os pacientes assistidos pelo SUS. Rev Bras Enferm 1999; 52(1):43-53.

22. Unglert CVS. Territorialização em sistemas de saúde. In: Mendes EV, organizador. Distrito sanitário. São Paulo: Hucitec; Rio de Janeiro: Abrasco; 1995. p. 221-235.

23. Ibope. Atendimento na unidades públicas de saúde. Seção Opinião Pública - Área: Análises e Índices $\$ 2000$. [site da Internet]. [acessado $2007 \mathrm{fev} 13$ ]. Disponível em: http://www.ibope.com.br/calandra Web/servlet/CalandraRedirect?temp $=6 \& \mathrm{proj}=$ PortalI

24. Kloetzel K, Bertoni AM, Irazoqui MC, Campos VPG, Santos RN. Controle de qualidade em atenção primária à saúde: I - A satisfação do usuário. Cad Saude Publica 1998; 14(3):623-628.

25. Brasil. Ministério da Saúde. Secretaria de Ciência, Tecnologia e Insumos Estratégicos. Aquisição de medicamentos para a assistência farmacêutica no SUS. Brasília: Ministério da Saúde; 2006. [acessado 2008 maio 8]. Disponível em: http://portal.saude.gov.br/portal/ arquivos/pdf/aquisicao_medicamentosfinal. pdf
26. Brasil. Ministério da Saúde. Secretaria de Políticas de Saúde. Departamento de Atenção Básica. Política Nacional de Medicamentos 2001. Brasília: Ministério da Saúde; 2001.

27. Gerschman S, Veiga L, Guimarães C, Ugá MAD Portela MC, Murat M, Barbosa P, Lemos S. Estudo de satisfação dos beneficiários de planos de saúde de hospitais filantrópicos. Cien Saude Colet 2007; 12(2):487-500

28. Pessoto UC, Heimann LS, Boaretto RC, Castro IE, Kayano J, Ibanhes LC, Junqueira V, Rocha JL, Barboza R, Cortizo CT, Martins LC, Luiz OC. Desigualdades no acesso e utilização dos serviços de saúde na Região Metropolitana de São Paulo. Cien Saude Colet 2007; 12(2):351-362.

29. Brasil. Ministério da Saúde. Carta dos direitos dos usuários da saúde: ilustrada. 2a ed. Brasília: Ministério da Saúde; 2007. (Série F, Comunicação e Educação em Saúde).

30. Gouveia GC, Souza WV, Luna CF, Souza Júnior PRB, Szwarcwald CL. Health care users' satisfaction in Brazil, 2003. Cad Saude Publica 2005; 21(Supl. 1):S109-S118.

31. Assis MMA, Villa TCS, Nascimento MAA. Acesso aos serviços de saúde: uma possibilidade a ser construída na prática. Cienc Saude Colet 2003; 8(3):815823.

32. Donabedian A. Evoluación de la calidad de la atención médica: investigaciones sobre servicios de salud - una antología. Washington: Organización Panamericana de la Salud; 1992. (Publicación Científica, 534).

33. Travassos C, Oliveira EXG, Viacava F. Desigualdades geográficas e sociais no acesso aos serviços de saúde no Brasil: 1998 e 2003. Cien Saude Colet 2006; 11(4):975-986.

Artigo apresentado em 08/10/2008

Aprovado em 27/11/2008

Versão final apresentada em 15/12/2008 